- Acute dental pain may be severe and result in large quantities of 'over the counter' (OTC) medications being taken by patients for symptomatic relief.

- Two cases of unintentional paracetamol overdose, arising as a result of dental pain, requiring anti-toxicity treatment are presented.

- Emphasis is placed on the description of the signs, symptoms and management of paracetamol overdose.

- A summary of paracetamol pharmacology is presented.

\title{
Paracetamol overdose as a result of dental pain requiring medical treatment - two case reports
}

\author{
M. B. M. Thomas, ${ }^{1}$ N. Moran, ${ }^{2}$ K. Smart ${ }^{3}$ and S. Crean ${ }^{4}$
}

\begin{abstract}
Two cases of unintentional paracetamol overdose are presented. Over a one month period these patients presented to an Accident and Emergency (ACEE) department with symptoms of paracetamol toxicity, following the ingestion of large quantities of analgesia for the self treatment of dental pain. In one case the patient had no access to a dentist. Both patients required admission under the care of the medical on-call team and required anti-toxicity treatment to prevent permanent liver injury. Subsequent referrals were made to the oral and maxillofacial surgery team who provided emergency dental treatment and advice on further dental care. This paper highlights the significant signs and symptoms of paracetamol overdose about which dental practitioners should be aware. It also describes the management principles required to prevent potentially life threatening liver damage. Discussion is also made of the potential impact on patients struggling to cope with pulpal pain without access to a general dental practitioner.
\end{abstract}

\section{INTRODUCTION}

Acute dental pain can be very severe, leading to significant patient distress. Sufferers may consume large quantities of over the counter (OTC) analgesics while waiting for appointments with general dental services, assuming they have access to a dentist at all. Paracetamol is easily available over the counter and not surprisingly is popular with those who are seeking symptom relief.

Paracetamol is a recognised cause of overdose that can result in a slow unpleasant death due to liver necrosis. It is often used in deliberate suicide

\footnotetext{
${ }^{1 *}$ SHO Oral and Maxillofacial Surgery, ${ }^{2}$ Locum Consultant Oral Surgeon, ${ }^{3}$ Locum Consultant Oral Surgeon, ${ }^{4}$ Consultant Oral \& Maxillofacial Surgeon, Oral and Maxillofacial Surgery Department, Prince Charles Hospital, Merthyr Tydfil, CF47 9DT

*Correspondence to: Matthew Thomas

Email:matthewbmthomas@hotmail.co.uk
}

\section{Refereed Paper}

Accepted 14 December 2006

DOI: $10.1038 /$ bdj.2007.583

${ }^{\circledR}$ British Dental Journal 2007; 203: 25-28 attempts; accounting for $9 \%$ of drug related suicides. ${ }^{1}$ In response to analgesic related mortality in the UK, legislation was introduced in 1998 to limit analgesic pack sizes. This resulted in a reduction in paracetamol related suicidal deaths by $22 \%$, in the year after the change in legislation. ${ }^{2}$ However even with these changes it is still possible to obtain large quantities of paracetamol. It is unsurprising that patients may be unaware of the maximum dose of paracetamol and the associated dangers of accidental over-consumption.

A search of the literature has revealed only a few reports of paracetamol overdoses in patients attempting to relieve dental pain. One report describes an unintentional paracetamol overdose, secondary to acute dental pain for which the patient did not require additional medical treatment ${ }^{3}$ while another describes a patient with liver failure secondary to paracetamol overdose arising from an attempt to relieve the symptoms of pericoronitis. ${ }^{4}$
Thus we present two cases of unintentional overdose of paracetamol in patients suffering acute dental pain, presenting over a one month period to an A\&tE department of a district general hospital. Both cases required treatment with paracetamol antidote.

\section{CASE REPORT 1}

A 20-year-old female student presented to the A\&tE department at 11pm over the Christmas period, complaining of toothache even though she had an appointment with her general dental practitioner (GDP) the following day. She admitted that in her efforts to ease her toothache, she had taken 16 paracetamol, 16 ibuprofen and two co-codamol (combined paracetamol and codeine phosphate doses) during the preceding 24 hours. There was no reported intent of self harm. Based on her weight of $40 \mathrm{~kg}$, this equated to a consumed paracetamol dose of $225 \mathrm{mg} / \mathrm{kg}$ and $80 \mathrm{mg} / \mathrm{kg}$ of ibuprofen, taken as staggered doses. Following consultation with Toxbase (National Poisons 


\begin{tabular}{|c|c|c|c|}
\hline & $\begin{array}{l}\text { Time since } \\
\text { ingestion }\end{array}$ & Signs & Symptoms \\
\hline Phase 1 & $<24$ hours & Pallor, malaise, vomiting, sweating & Anorexia, nausea, vomiting \\
\hline Phase 2 & $24-48$ hours & Tachycardia, hypotension & Upper abdominal pain \\
\hline Phase 3 & 3-4 days & Jaundice, bleeding, encephalopathy & GI bleeding \\
\hline Phase 4 & 4-11 days & $\begin{array}{l}\text { Recovery or death depending on } \\
\text { severity of damage }\end{array}$ & \\
\hline
\end{tabular}

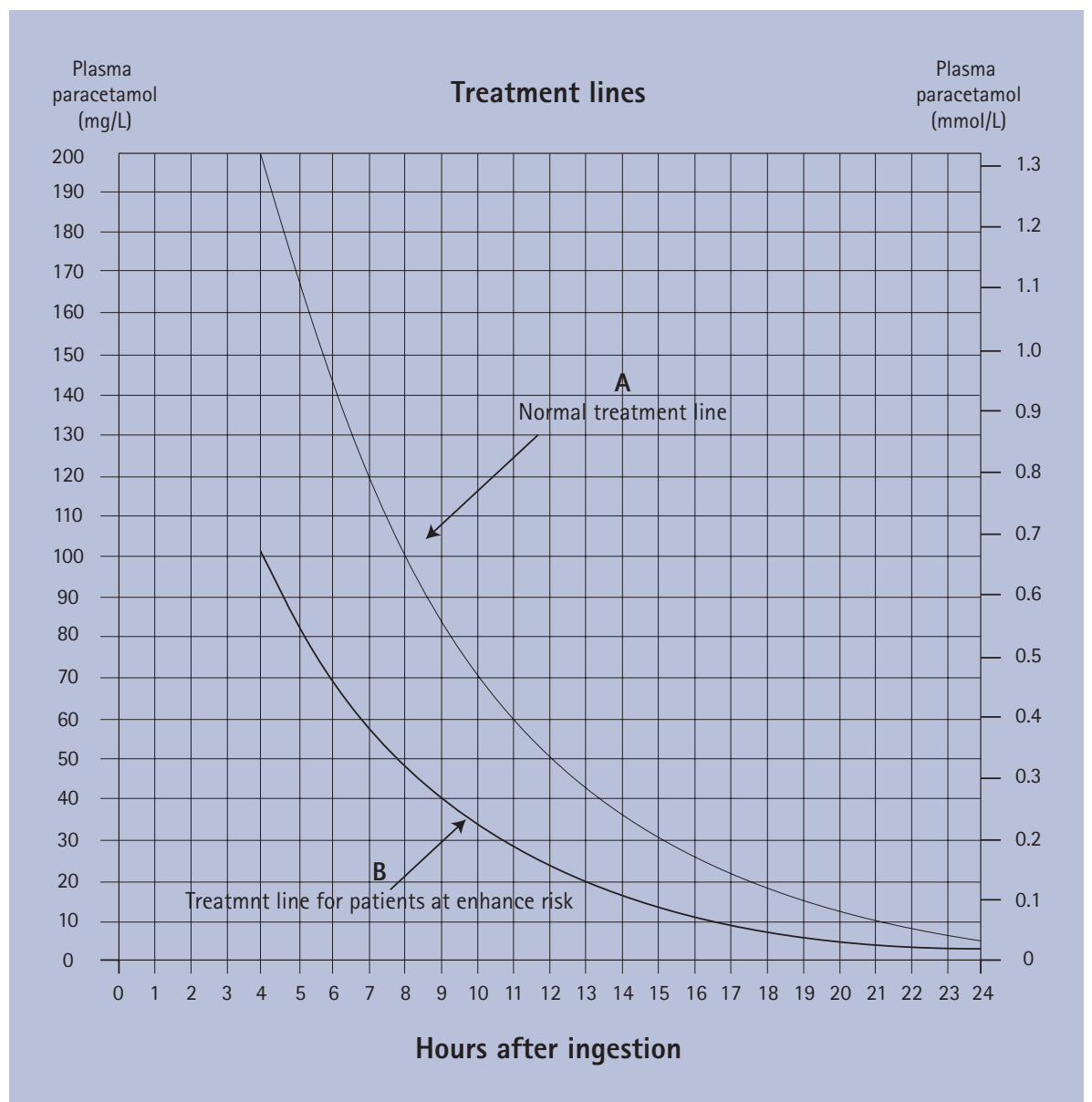

Fig. 1 Adapted from 'guidelines for the management of acute paracetamol overdosage' - http://www.pharmweb.net/pwmirror/pwy/paracetamol/chart.html

Information Database), $\mathrm{N}$ acetyl-cysteine (NAC) treatment (Parvolex $\left.{ }^{\circledR}\right)$ was instigated to treat the threat posed by the paracetamol dosage. Blood investigations were performed and clinical observations noted. No action was required for the quantities of ibuprofen taken.

As the patient was still in substantial pain the on-call SHO in oral and maxillofacial surgery was requested to assess the patient. She reported a 24 hour history of unbearable toothache from the lower left quadrant. The patient was normally fit and
(INR) and paracetamol levels were recorded as a baseline. Paracetamol levels were monitored regularly and shown to decrease from $35.5 \mu \mathrm{g} / \mathrm{ml}$ to $12 \mu \mathrm{g} / \mathrm{ml}$ over two hours of Parvolex ${ }^{\circledR}$ treatment.

The patient's dental pain returned the following day and so with the agreement of the medical team she was taken to the oral and maxillofacial department for extirpation of the pulp tissue and dressing of the lower left second permanent molar. She was advised to return to her GDP for continued treatment.

Repeat blood investigations were normal and her INR was 1.0 suggesting that liver damage was unlikely. Her dental pain had subsided enough for her to be discharged from the hospital but with arrangements for follow-up.

\section{CASE REPORT 2}

A 20-year-old male attended the ActE department, at $10.56 \mathrm{pm}$ one midweek evening, complaining of toothache from which he had been suffering for some months. He was not registered with a dentist. He informed the staff that he had taken 10 paracetamol tablets that night. When called by the doctor he had left the department. The patient returned four hours later by ambulance by which time he had consumed a further 10 paracetamol tablets and was vomiting repeatedly. At this point he admitted to the casualty doctor to taking approximately 60 tablets over the last three days. This was calculated as approximately $428 \mathrm{mg} / \mathrm{kg}$ as a staggered dose.

The patient was usually fit and well and there was no intent on self harm. On examination the patient was in distress, complaining of abdominal pain with tenderness of the upper right quadrant and vomiting bile products. The abdomen was soft on palpation but with some evidence of guarding. A provisional diagnosis of staggered dose paracetamol overdose and possible liver failure was made. The paracetamol antidote (NAC, Parvolex ${ }^{\circledR}$ ) was administered along with opioid analgesia. Appropriate baseline blood investigations were carried out which comprised full blood count, urea and electrolytes assay, liver function tests, INR, arterial blood gases, plasma paracetamol levels. The patient was transferred to the medical ward.

Blood investigations taken on presentation showed elevation of white cell count suggestive of active infection, elevation of 
bilirubin and an INR of 1.1. The plan was to repeat INR and LFT daily to see if any permanent liver damage had developed.

The on-call SHO in oral and maxillofacial surgery was called to assess the dental pain. Clinical and radiographic examination revealed a grossly carious upper right first permanent molar with buccal sulcus tenderness consistent with a developing dentoalveolar abscess. The tooth was extracted 24 hours after admission, after liver damage and associated increased bleeding tendency could be ruled out by repeat blood investigation. The patient was discharged the next day as INR and LFT had been stable for 48 hours and the paracetamol plasma concentration had dropped from 25.9 $\mu \mathrm{g} / \mathrm{ml}$ on admission to $<10.0 \mu \mathrm{g} / \mathrm{ml} 24$ hours later.

\section{DISCUSSION}

These two cases demonstrate the potential for serious health problems in patients self medicating OTC analgesics in an attempt to gain relief from dental pain. It is essential for patient welfare that they have access to medical advice to guide them through this stage until definitive care can be arranged. The first of the patients presented was registered with a dentist but was unable to secure an appointment until the following day. The second of the patients presented was not registered with a dentist at all, which raises intriguing issues in these turbulent times of diminishing numbers of NHS dentists and the nationwide problem of access to dental care. It may be that both these patients were unaware of the risks of excessive paracetamol consumption, something shown in previous papers, ${ }^{3}$ making guidance from health care professionals paramount.

Both these patients had ingested sufficient doses of paracetamol to require medical care. The issue was detected by careful questioning in the casualty department but the lessons to be learned from these clinical episodes have special relevance to all in the dental profession. Primary among these is: the need to recognise at risk patients from the history and clinical examination and the pharmacology behind this effective but potentially hazardous drug.

Paracetamol (acetaminophen in the USA) is a very effective over the counter analgesic for the treatment of dental pain. It is an alanine derivative with primarily analgesic and antipyretic properties. The drug is well absorbed from the small intestine after oral ingestion with peak plasma concentrations within 60 minutes and a half life of two hours. ${ }^{5}$ Paracetamol is metabolised in the liver by a process called conjugation. Its mechanism of action is unclear but it is a less potent inhibitor of the cyclooxygenase system (COX) than conventional non-steroidal anti-inflammatories (NSAIDS), accounting for its minimal anti-inflammatory effect. It is active at inhibiting brain prostaglandin (PGE1 and PGE2) synthesis responsible for the analgesic effect. Inhibition of PG synthesis also occurs within the hypothalamus resulting in the antipyretic effects.

Side effects of paracetamol overdose include skin rashes and white blood cell disorders, but more seriously, hepatotoxicity of overdose. At normal doses paracetamol is broken down in the liver to glucuronide and sulphate conjugates. A small proportion is also converted by the microsomal p450 oxidation chain to a reactive metabolite N-acetyl-pbenzoquinone imine (NAPBQI). This metabolite is conjugated with glutathione to form cysteine and mercapturate conjugates. In overdose, available supplies of glutathione are exhausted and NAPBQI accumulates contributing to hepatocellular necrosis. N-acetyl cysteine supplements, eg Parvolex ${ }^{\circledR}$ acts as a source of thiol groups that may be used in the synthesis of additional glutathione, hopefully reducing hepatocellular damage.

As little as $10-15 \mathrm{~g}$ (20-30 tablets) or $150 \mathrm{mg} / \mathrm{kg}$ of paracetamol taken within 24 hours may cause severe hepatocellular necrosis, acute liver damage and less frequently renal tubular necrosis. ${ }^{6}$

Many patients suffering from acute dental pain may be unaware of the number of analgesic preparations they have consumed in their pursuit of symptom relief. The problem of overdose is compounded by the fact that a significant number of cases exhibit no obvious signs during the first 24 hours following ingestion. It is therefore important that both primary care and hospital practitioners are aware of the signs and symptoms that may indicate a patient is at risk of a paracetamol overdose (Table 1). Since many of the early signs and symptoms are non-specific a thorough drug history is essential for anyone self medicating with dental pain so that an urgent transfer to a medical unit can be made.

Early treatment of overdose is essential. Gastric lavage will prevent further absorption if provided within the first hour of ingestion. ${ }^{5} \mathrm{~N}$-acetylcysteine (NAC eg Parvolex ${ }^{\circledR}$ )) protects the liver if infused within 24 hours of ingestion. Parvolex ${ }^{\circledR}$ conjugates with the accumulating NAPBQI protecting cells from damage. Patients that require treatment can be identified by the plasma paracetamol concentration related to the time of ingestion (Fig. 1). This also applies to those who have consumed a single large dose at a known time. This treatment should continue until plasma-paracetamol concentration significantly reduces. This concentration can be plotted on a paracetamol treatment graph. Those with plasma-paracetamol concentration above the treatment line receive $n$-acetyl cysteine. Minimal hepatic impairment can be anticipated when the plasma concentration is less than $120 \mathrm{\mu gml}^{-1}$ at four hours or $30 \mathrm{\mu gml}^{-1}$ at 12 hours. Plasmaparacetamol concentration may be difficult to interpret when paracetamol has been ingested over several hours. If there is doubt patients should receive the antidote.

$\mathrm{N}$-acetylcysteine is given intravenously, initially as $150 \mathrm{mg} / \mathrm{kg}$ in $200 \mathrm{ml}$ of 5\% dextrose over 15 minutes, then 50 $\mathrm{mg} / \mathrm{kg}$ in $500 \mathrm{ml}$ of $5 \%$ dextrose over four hours then $100 \mathrm{mg} / \mathrm{kg}$ in $1000 \mathrm{ml}$ of 5\% dextrose over 16 hours. The overall management of the patients can be summarised as follows, adapted from the NPIS guidelines: ${ }^{7}$

1. Question the patient and others on the number of paracetamol tablets taken and the time of ingestion as this is critical in determining treatment need from Figure 1

2. Blood investigations should be requested including INR, plasma bicarbonate, urea and electrolytes, plasma creatinine and LFTs. Vital signs should be recorded regularly

3. Plasma paracetamol levels should be recorded four hours after ingestion to allow for complete absorption and distribution of the drug

4. Compare these levels to the graph in Figure 1 depending on whether patient is deemed high risk or not 5. If dose consumed is less than $150 \mathrm{mg} /$ $\mathrm{kg}$ and plasma paracetamol concentration is below treatment line patient 
does not need treatment

6. If plasma concentration is above treatment line start treatment with NAC

7. Those patients who have taken staggered doses should be treated anyway as the graph shown in Figure 1 is meaningless as time of ingestion is continuous

8. Those who have received NAC can be discharged 24 hours after last ingestion provided they have completed the course and are asymptomatic and INR, ALT and plasma creatinine are normal.

Patients suffering from dental pain are more likely to consume high levels of paracetamol as a staggered dose rather than single large doses with intention to cause self harm. The guidelines show that in these patients NAC treatment should be started immediately as the graph of plasma paracetamol concentration by time is meaningless.

These two cases highlight the benefits of dentists educating their patients on the safe use of analgesia, especially paracetamol, but also emphasises the need for such health professionals to be totally familiar with the drugs their patients are taking. Many over the counter analgesics (OTC) contain paracetamol such as Panadol $^{\circledR}$ or paediatric medications such as Calpol ${ }^{\circledR}$ and Disprol ${ }^{\circledR}$. Parents may be unaware of the sources of paracetamol that may result in accidental overdose of their child. Several OTC medications are compound in nature combining opioid analgesia with paracetamol such as Co-codamol in Solpadol ${ }^{\circledR}$ and Kapake ${ }^{\circledR}$. The consumption of additional paracetamol with these medications could be catastrophic. It is also important to bear in mind that several commonly used medications such as 'cold and flu' tablets contain significant amounts of paracetamol, as much as $1000 \mathrm{mg}$ eg Lemsip Max cold and $\mathrm{flu}^{\circledR}$. Likewise anti-migraine tablets such as Migraleve $^{\circledR}$ combine paracetamol with buclizine hydrochloride. Unless patients carefully examine the packaging of these OTC medications they may be unaware of this important fact. If such patients suffer from dental pain and take additional paracetamol they may unknowingly approach toxic doses. Dentists therefore need to be aware of all possible sources of paracetamol their patients may be taking, prior to advising on appropriate analgesia for dental pain.

The authors would like to thank Dr A. Hutchings from the Toxicology Department Llandough Hospital, Cardiff for his permission to reproduce the graph in Figure 1.

1. Hawton K, Simkin S, Deeks J. Co-proxamol and suicide: a study of national mortality statistics and local non-fatal self poisonings Br Med J 2003; 326: 1006-1008.

2. Hawton K, Simkin S, Deeks J et al. UK legislation on analgesic packs: before and after study of long term effect on poisonings. Br Med J 2004; 329: 1076-1079.

3. Dodd M D, Graham C A. Unintentional overdose of analgesia secondary to acute dental pain. Br Dent J 2002; 193: 211-212.

4. Sivaloganathan K, Johnson P A, Bray G P et al. Pericoronitis and accidental paracetamol overdose, a cautionary tale. Br Dent J 1993: 174: 69-71.

5. Seymour J, Meechan J, Yates S. Pharmacology and dental therapeutics. (3 ${ }^{\text {rd }}$ edn) pp 92-93. Oxford: Oxford University Press, 1999

6. British national formulary. 50 September 2005.

7. NPIS guidelines on paracetamol overdose. Revised edition 2003. 